\title{
Stage IIIC Esophageal Adenocarcinoma AJCC v7
}

National Cancer Institute

\section{Source}

National Cancer Institute. Stage IIIC Esophageal Adenocarcinoma A/CC v7. NCI

Thesaurus. Code C87786.

Stage IIIC includes: (T4a, N1-2, M0, Any G); (T4b, Any N, M0, Any G); (Any T, N3, M0, Any G). T4a: Resectable tumor invading pleura, pericardium, or diaphragm. T4b: Unresectable tumor invading other adjacent structures, such as aorta, vertebral body, trachea, etc. N1: Metastasis in 1-2 regional lymph nodes. N2: Metastasis in 3-6 regional lymph nodes. N3: Metastasis in seven or more regional lymph nodes. M0: No distant metastasis. (AJCC 7th ed.) 